\title{
Bronchial carcinoid presenting as Cushing's syndrome: A challenging diagnostic conundrum
}

Authors: Yew Wen Yap ${ }^{1}$, Aftab Ahmad ${ }^{1}$, Dushyant Sharma

\section{Introduction}

Localising the source for Cushing's syndrome can be a challenge, when the investigations being performed are limited in its sensitivities and specificities. This case report explores these challenges with investigating a patient with clinical and biochemical features of Cushing's syndrome.

\section{Case}

A 70 year old female presented to the endocrine outpatient clinic with lethargy, fatigue, poor sleeping patterns, one stone of weight gain and intermittent headaches for twelve months. Additionally, she had difficulties standing up from sitting and was unable to walk up the stairs. She had six weeks of dry cough with an eight year history of generalised muscle aches.

Her past medical history includes hypothyroidism, depression, hypertension, hypercholestrolaemia, hysterectomy for bladder prolapse and pancreatitis. She was a non-smoker and was a retired shop assistant. Her exercise tolerance was limited to a few hundred yards. Her regular medications includes Ramipril, Citalopram, Omeprazole, Irbesartan, Levothyroxine and Co-codamol.

On physical examination, she had telangiectasia across both cheeks, pink striae across her abdomen and flanks with proximal muscle wasting. Her blood pressure was $215 / 112 \mathrm{mmHg}$. A differential diagnosis of Cushing's syndrome was suspected. Her $\mathrm{HbA} 1 \mathrm{c}$ was $41 \mathrm{mmol} / \mathrm{l}$.

\section{Results}

1a) $1^{\text {st }} 24$ hour urinary cortisol: $478 \mathrm{nmol} /$ 24 hours.

b) $2^{\text {nd }} 24$ hour urinary cortisol: $326 \mathrm{nmol} /$ 24hours. ( Reference: $<165 \mathrm{nmol} / 24$ hours)

\section{2) Overnight dexamethasone suppression:}

Baseline ACTH: 33nmol//, cortisol $731 \mathrm{nmol} / \mathrm{I}$ Post overnight dexamethasone suppression: cortisol: $131 \mathrm{nmol} / \mathrm{l}$ (normal: $<50 \mathrm{nmol} / \mathrm{l}$ )
3) High dose dexamethasone suppression (HDDST):

Baseline: $131 \mathrm{nmol} / \mathrm{l}, \mathrm{ACTH}: 20 \mathrm{pmol} / \mathrm{I}$ Post HDDST 48 hours later: < 50nmol/l

4) MRI pituitary: Normal pituitary gland with no evidence of adenoma.

5) CT chest abdomen \& pelvis: No evidence of any malignancy.

6) Inferior petrosal sinus sampling: no difference between the ratios on both sides of the inferior petrosal sinus and peripheral samples, excluding a pituitary source for excessive ACTH production.

\begin{tabular}{|l|l|l|l|}
\hline $\begin{array}{l}\text { Time } \\
(\mathrm{min})\end{array}$ & $\begin{array}{l}\text { Left } \\
(\mathrm{pmol} / \mathrm{L})\end{array}$ & $\begin{array}{l}\text { Right } \\
(\mathrm{pmol} / \mathrm{L})\end{array}$ & $\begin{array}{l}\text { Peripheral } \\
(\mathrm{pmol} / \mathrm{L})\end{array}$ \\
\hline 0 & 37 & 27 & 26 \\
\hline 5 & 32 & 33 & 30 \\
\hline 10 & 30 & 30 & 32 \\
\hline 16 & 30 & 29 & 32 \\
\hline
\end{tabular}

7) MRI NM Ga 68 DOTANAC scan: no abnormal somatostatin receptor positive lesions identified initially.

8) MRI Thorax: Nodule in the left lower lobe lung: $1.2 \mathrm{~cm}$

Radiologist re-review NM Ga68 DOTANAC scan :small focus of trace activity posterior to a $7 \mathrm{~mm}$ nodule in the left lung base which revealed some mild somatostatin receptor positivity. Corresponded to lesion on Thoracic MRI. Inter and intra-observer variability in identifying such a small lesion.

9) Further MRI NM MIBG lodine 123 No increase in tracer activity in small pulmonary nodule.

10) NM Whole Body FDG PET CT: $1.2 \mathrm{~cm} X$ $1.1 \mathrm{~cm}$ nodule within left lower lobedemonstrating low activity.

She went on to have a Video Assisted Thoracoscopy assisted left lower lobectomy and this was identified as a $11 \mathrm{~mm}$ nodule, cream- coloured on histology.

This was classified as a typical ACTHproducing carcinoid tumour which was staged as T1a NO MO

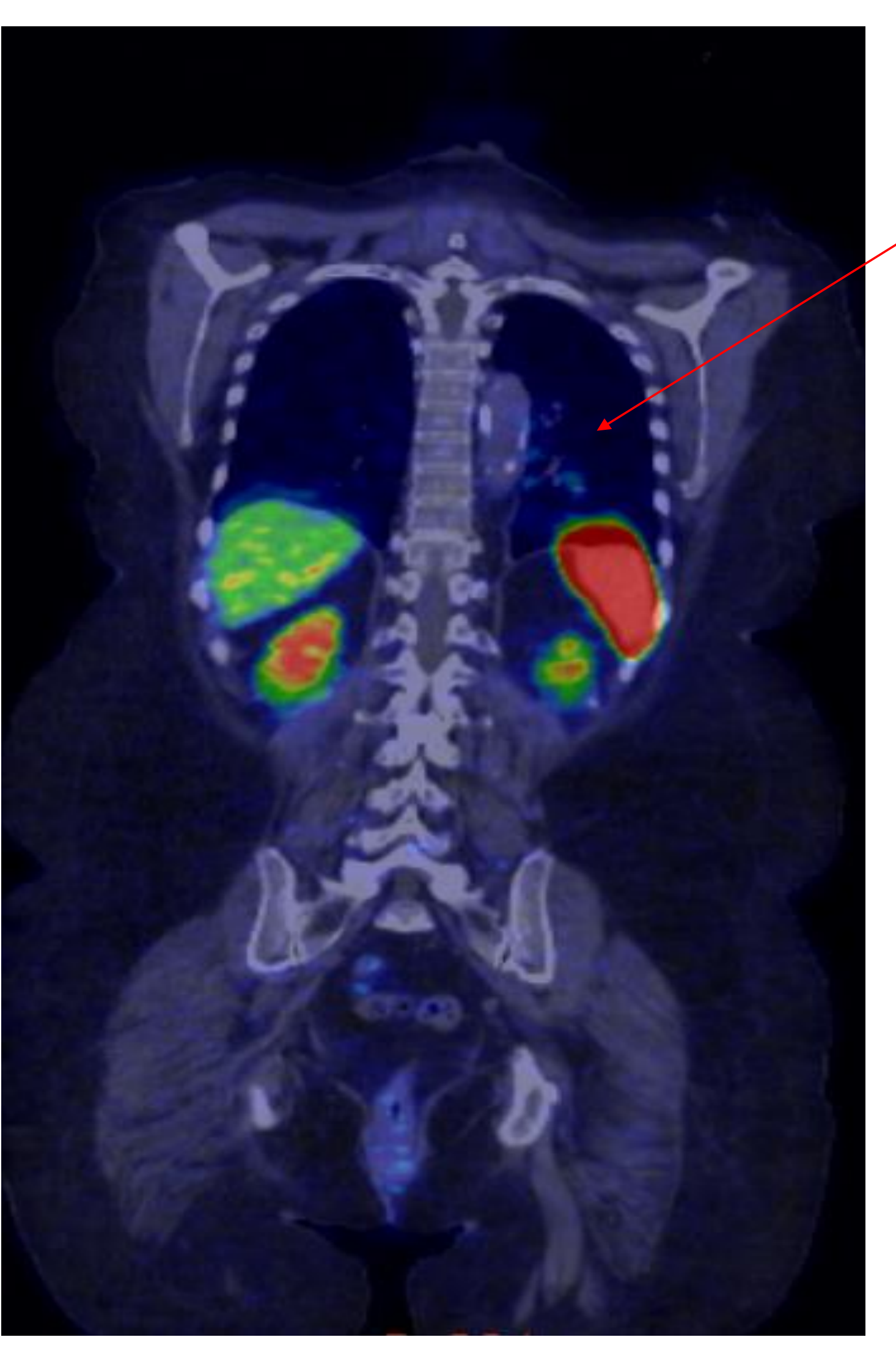

$1.2 \mathrm{~cm} \times 1.1 \mathrm{~cm}$ nodule within the left lower lobe as identified with the red arrow on NM Ga 68 DOTANOC PET CT \& on FDG PET CT with the blue arrow.

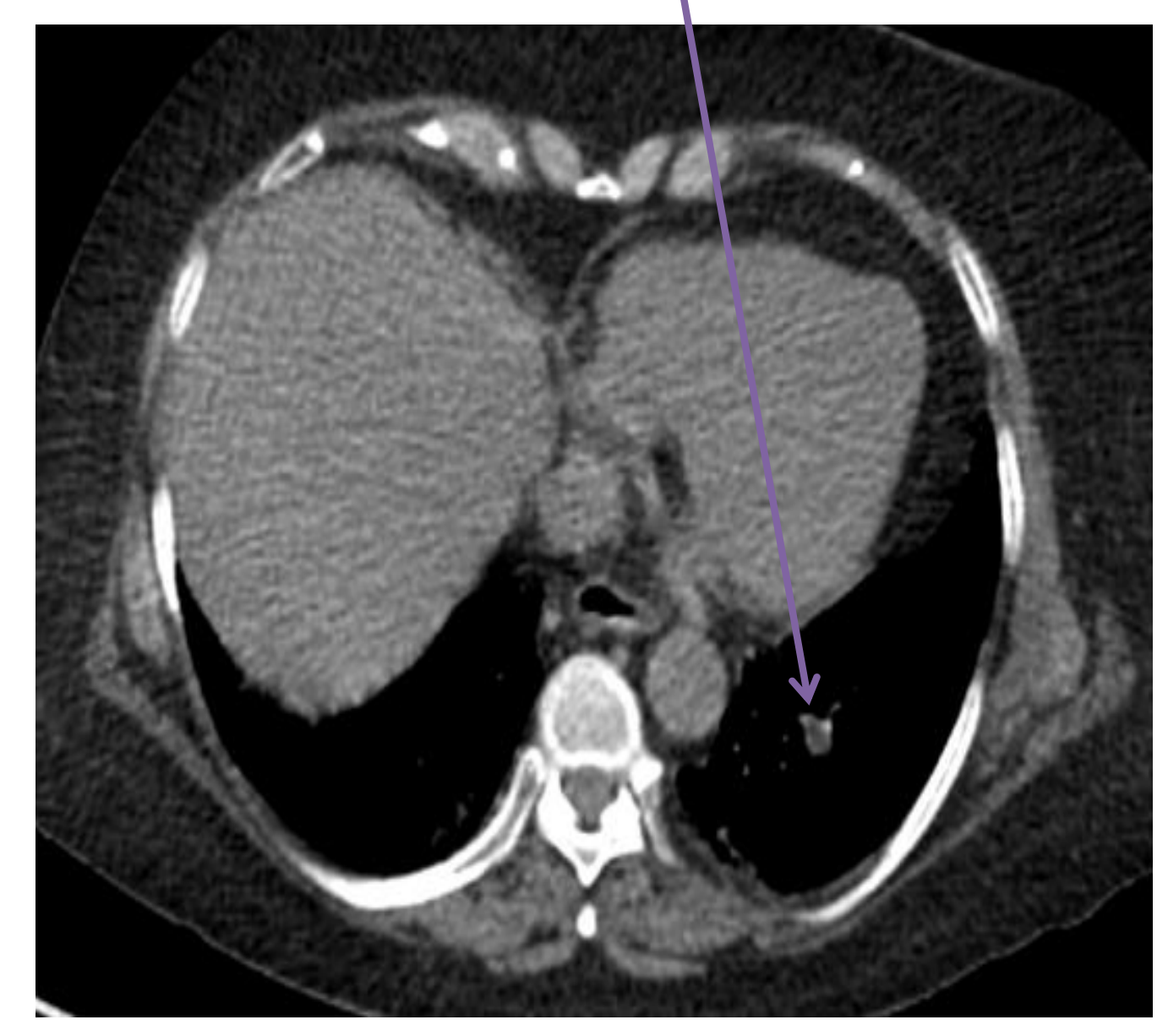

\section{Discussion}

Ectopic ACTH production accounted for $10-15 \%$ of Cushing's syndrome and Ectopic $\mathrm{CRH}$ secretion for less than $1 \%{ }^{1}$. In the group of ectopic ACTH, only about $1-5 \%$ of Bronchial Neuroendocrine tumours were associated with ectopic ACTH secretion ${ }^{2,3}$.

The use of the High dose dexamethasone suppression test was found to be limited as with this case, as noted in a case series that up to $83 \%$ of ectopic ACTH producing bronchial tumours demonstrated suppression on HDDST, making the diagnosis very difficult ${ }^{4}$. Additionally, it was noted that with the size of these carcinoid tumours, they can also easily elude imaging on CT Chest ${ }^{5}$. Thus, we recommend the use of functional imaging such as the DOTANAC scans for further correlation, together with Inferior Petrosal Sinus sampling to differentiate between a pituitary and an ectopic source of Cushing's syndrome in indeterminate cases of elevated ACTH levels.

\section{Conclusion}

The limitations in investigations for Cushing's syndrome should be taken into account when investigating patients for this condition. We recommend that when biochemical testing becomes inconclusive, it should prompt further investigations such as MRI Pituitary and Inferior petrosal sinus sampling to rule out a pituitary source. Additionally, specialist radiological modalities such as NM Ga68 DOTANAC scanning should be sought to identify the causative lesion, especially when ectopic sources are suspected. 